\title{
New Imaging Methods of Coronary Arteries in Acute Coronary Syndromes
}

\author{
Ota Hlinomaz ${ }^{1,2}$, Ladislav Groch ${ }^{1,2}$, Jan Sitar ${ }^{1,2}$, Michal Rezek ${ }^{1,2}$, Jiří Seménkaa, \\ Martin Novák ${ }^{1,2}$, Nikolay Penkov ${ }^{2}$
}

${ }^{1}$ Department of Cardioangiology, ICRC, St. Anne University Hospital, Brno, Czech Republic

2 SBALK Varna, Bulgarian Cardiac Institute, Bulgaria

\section{ABSTRACT}

Coronary angiography is still the most widely used method for the assessment of lumen of coronary arteries and for diagnosis and treatment of coronary artery disease. New imaging modalities of coronary arteries play an increasing role in interventional cardiology. Intravascular ultrasound (IVUS) is the oldest technology, however due to its high tissue penetration remains very important for imaging of left main coronary artery and saphenous vein grafts. IVUS was used in many clinical trials and clinical experience with it is huge. Optical coherence tomography (OCT) is a new, very fast developing method. It has ten times higher axial resolution than IVUS. It gives us the opportunity to assess the inner structures of coronary artery wall, to evaluate the characteristics of atherosclerotic plaques, quality of stent implantation and its healing. It helps us to find the culprit lesion of acute coronary syndrome in some cases, to diagnose the cause of stent thrombosis, and to evaluate stent apposition which has a direct relation to prognosis. We use it to perform complex percutaneous coronary interventions and after heart transplantation to diagnose the vascular graft disease. We strongly believe that OCT is important for the assessment of plaque instability and patient's prognosis. Near infrared spectroscopy combined with IVUS can distinguish fibrous from lipid core plaques. Lipid core burden index is in relation to a risk of periprocedural myocardial infarction and to prognosis. It is the only method which can sufficiently detect the amount of lipids in coronary wall.

Keywords: optical coherence tomography, near infrared spectroscopy, intravascular ultrasound, atherosclerotic plaque

\section{ARTICLE HISTORY}

Received: 20 September, 2015

Accepted: 20 November, 2015

\section{CORRESPONDENCE}

\section{Ota Hlinomaz}

Department of Cardioangiology International Clinical Research Center (ICRC) - St. Anne's University Hospital in Brno, Masaryk's University Pekarska 53, 65691 Brno, Czech Republic

Email: ota.hlinomaz@fnusa.cz

\section{INTRODUCTION}

Coronary angiography is still the most widely used method for the assessment of lumen of coronary arteries and for diagnosis and treatment of coronary artery disease. New imaging modalities of coronary arteries play an increasing role in interventional cardiology. Intracor- onary ultrasound (IVUS) is the oldest technology. Due to high tissue penetration it is very important for imaging of left main coronary artery and saphenous vein grafts. IVUS was used in many clinical trials and clinical experience with it is huge. Optical coherence tomography (OCT) and near infrared spectroscopy (NIRS) are novel imag- 
ing modalities of coronary arteries. Especially OCT has a widespread use in many patients with coronary artery disease now.

\section{OPTICAL COHERENCE TOMOGRAPHY}

Optical coherence tomography (OCT) is a new, very fast evolving method in the evaluation of the internal lumen and the wall of coronary arteries. Its principle is similar to intracoronary ultrasound, which uses sound reflections. OCT uses a light source of a wavelength 1300 $\mathrm{nm}$, which is near infrared light. Light emitted from the catheter is reflected by the microstructures in biological tissues and returns to the detector, which analyzes the changed wavelength of the light. Laser source is used by last OCT devices, we're talking about the so-called optical frequency domain imaging (OFDI). First OCT systems emerged in the early '90s of the last century and their use in imaging of the retina and blood vessels was assumed [1]. About ten years ago the first generation OCT was utilized only in some centers in restricted indications and only for research purposes. The need for temporary occlusion of the coronary artery by a baloon fixed to the proximal part of the OCT catheter was its main limitation. As blood elements significantly worsen the image quality, the distal lumen of the coronary artery has to be flushed with saline and thereby the presence of red blood cells between the catheter and the vessel wall at the time of recording is minimized. These were the so-called occlusive systems. The launch of non-occlusive systems several years ago was a real revolution in the market. There are two companies producing the second generation OCT. The first is St. Jude Medical, Inc. with $\mathrm{C}_{\mathrm{XR}}$ OCT catheter, the second is Terumo with a similar system. Monorail OCT catheters are very user friendly and allow us to obtain a very good record in a few seconds. They are very flexible and can be introduced into relatively tortuous parts of coronary arteries. Axial resolution of OCT, which depends on the width and wavelength of the light beam, is $12-15$ $\mu \mathrm{m}$, which is a clear advantage in comparison with intravascular ultrasound (IVUS). Lateral resolution influenced primarily by the optical system of the catheter is about $25 \mu \mathrm{m}$. High resolution is unfortunately at the expense of depth entry (tissue penetration), which is smaller than with IVUS. It is caused by reduction of intensity of the light beam as it penetrates the tissues, thus good elimination of blood in the catheter during recording is absolutely necessary $[2,3]$.

The comparison between OCT and IVUS shows that OCT is a more sensitive method for imaging of inner layers of coronary arteries and of implanted coronary stents, but is not suitable for examination of distant structures of coronary wall, e.g. for evaluation of coronary artery remodeling. Therefore devices and catheters that combine both methods or even provide data about functional significance of studied stenoses (fractional flow reserve - FFR) are in development. It should be however noted, that the vast majority of trials evaluating coronary arteries or results of PCI with stent implantation were performed with IVUS, which is therefore in many centers still used as an assistant device during PCI. OCT criteria for optimal stent expansion or prognostic indicators have not yet been determined. OCT is certainly the future, but it must be validated in routine clinical practice.

OCT helps to assess the cause and extent of coronary artery disease, which is important not only for clinical practice but also for many research projects evaluating the process of atherosclerosis and its medical treatment. OCT can be an integral part of complex PCI procedures with stent implantation. Like any surgical intervention, coronary stent implantation has its drawbacks and risks, and stent thrombosis is one of them. Early and late stent thromboses are distinguished. Early stent thrombosis occurs within 7 days after implantation and is caused mostly by insufficient expansion of the stent. In these cases the term "stent malapposition" is used. The status of the

TABLE 1. Comparison of OCT and IVUS devices [4]

\begin{tabular}{lcc}
\hline & OCT $\left(\mathbf{C 7}_{\mathbf{X R}}\right)$ & IVUS \\
\hline Wavelength & $1.3 \mu \mathrm{m}$ & $20-45 \mathrm{MHz}$ \\
Axial resolution & $12-15 \mu \mathrm{m}$ & $100-200 \mu \mathrm{m}$ \\
Lateral resolution & $20-40 \mu \mathrm{m}$ & $200-300 \mu \mathrm{m}$ \\
Frame/s (frame rate) & 100 & 30 \\
Pullback speed & $20 \mathrm{~mm} / \mathrm{s}$ & $0.5-1.0 \mathrm{~mm} / \mathrm{s}$ \\
Max. scan diameter & $9.7 \mathrm{~mm}$ & $15 \mathrm{~mm}$ \\
Tissue penetration & $1.0-2.5 \mathrm{~mm}$ & $10 \mathrm{~mm}$ \\
\hline
\end{tabular}


coronary artery before stent implantation (e.g. presence of thrombus or severe calcifications), the quality of antithrombotic therapy or a local reaction of the vessel to the implanted stent also play an eminent role. Late stent thrombosis ( $>7$ days after the procedure) is caused by insufficient healing of the stent into the vessel wall. Stent thrombosis, anytime it occurs, is always a very serious condition and life-threatening for the patient. Mortality of patients with stent thrombosis is very high, reaching about $50 \%$. Reduction of occurrence of stent thrombosis must be our goal. We have to define the risk indicators of stent thrombosis using the most sensitive method - optical coherence tomography, which allows us to identify the most vulnerable patients. OCT can be used to determine the appropriate length of the stent. It allows us to measure the diameter of coronary artery in a place where it is still healthy, which helps us to choose the appropriate size of the stent. OCT performed immediately after stent implantation is very useful for visualization of the relationship between the stent and the vessel wall, to determine the extent of potential inadequate stent apposition and thus provide the interventional cardiologist additional information for decision about stent postdilatation. OCT performed several weeks or months after stent implantation tells us about the ongoing healing

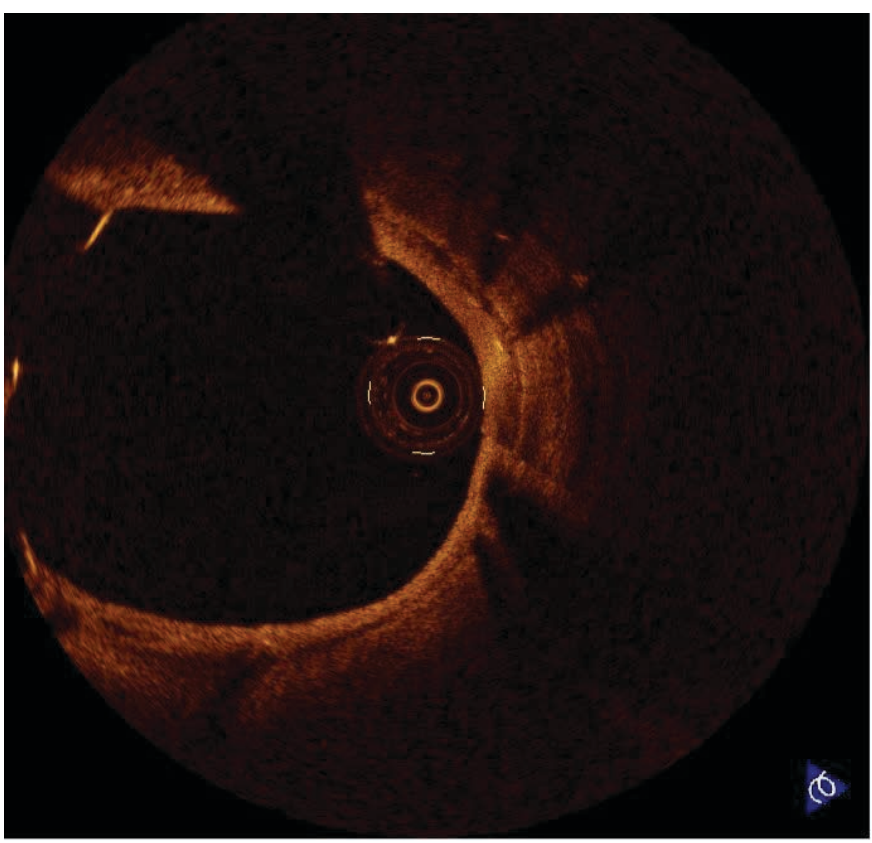

FIGURE 1. Bare metal stent implanted to the left main - left anterior descending artery (LAD) over left circumflex artery (LCX) without final kissing dilatation. OCT after 1 year. Small neointimal proliferation in segments $0-7$, free stent struts at the ostium of LCX (segments 9-11). process, about the coverage of the stent by tissue, which may be related to the prognosis of the patient. This is particularly important in the evaluation of drug eluting stents (DES), which on one side considerably reduce the incidence of restenosis, and on the other side there are frequent reports of their late thrombosis. It is assumed that the coverage of the stent by small amount of tissue (stent coverage) after several months has a favorable prognostic impact.

\section{OUR EXPERIENCE}

The authors examined by OCT 124 patients from January 2010 till December 2013. Seventy-six of them (61\%) had a diagnosis of chronic ischemic heart disease, 38 (31\%) acute myocardial infarction without ST segment elevation or unstable angina pectoris and 10 (8\%) ST segment elevation myocardial infarction. The indications for OCT were: to evaluate the stent apposition and stent healing, stent thrombosis, complex PCI procedures, to define the culprit lesion in patients with acute coronary syndrome, after heart transplantation. In total we have checked 151 stents, 36 (24\%) were drug eluting stents (DES), 57 (38\%) were stents with rapid endothelization and $58(38 \%)$ were bare metal stents (BMS).

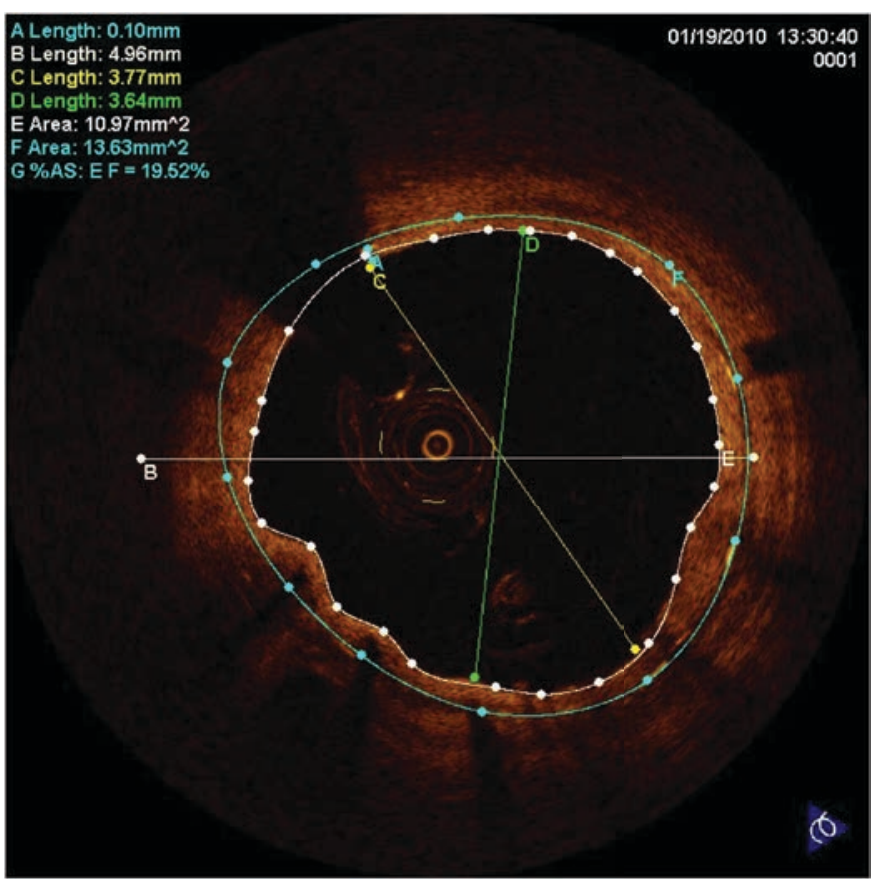

FIGURE 2. Similar to IVUS, OCT can measure the inner lumen of coronary arteries and determine the significance of coronary stenosis. OCT six months after implantation of a drug eluting stent (DES). The area of inner lumen is $11.0 \mathrm{~mm}^{2}$, the area stenosis is $19.5 \%$. 


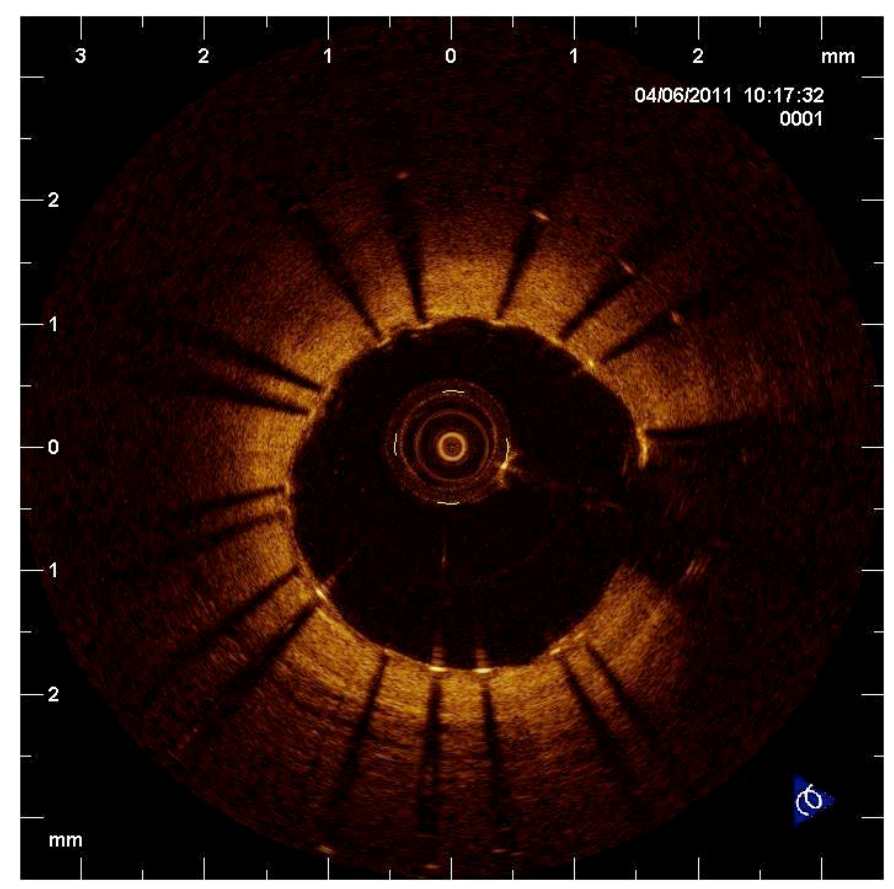

FIGURE 3. Stent with rapid endothelization $3.0 \mathrm{~mm} 18 \mathrm{~mm}$ postdilated with noncompliant balloon catheter $3.5 \mathrm{~mm} 14 \mathrm{~mm}$ to $16 \mathrm{~atm}$. Middle part of stent after 14 days. We can see the perfect apposition of the stent and start of healing.

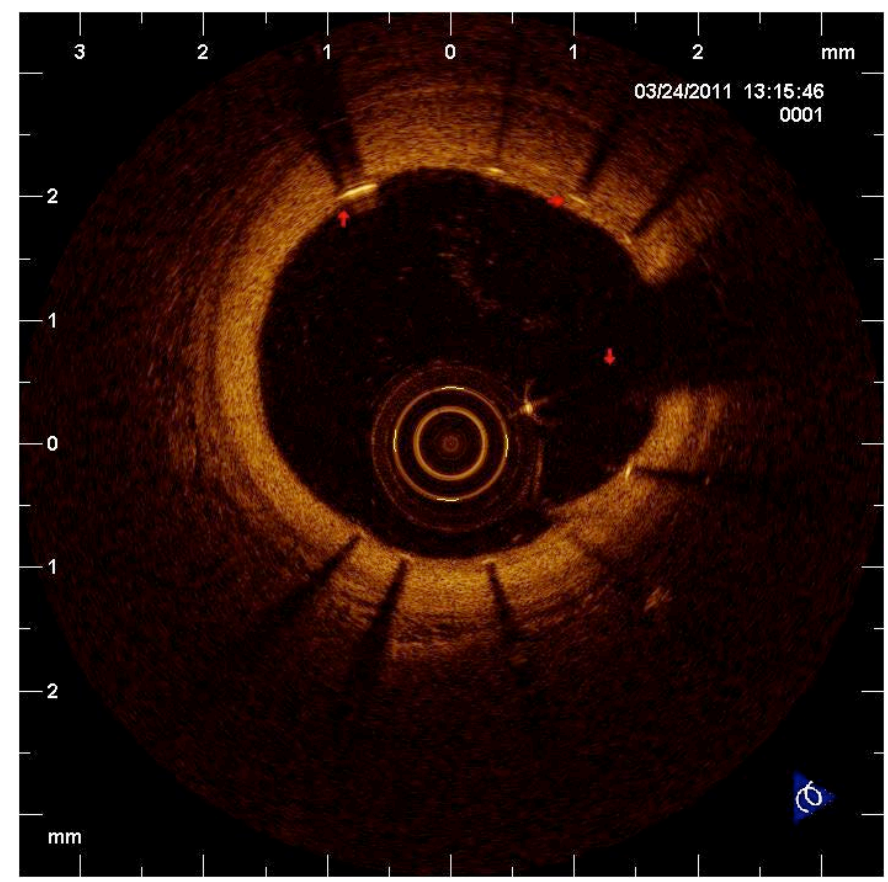

FIGURE 5. Good healing of the stent with rapid endothelialization $(3.0 \mathrm{~mm} 18 \mathrm{~mm}$ ) dilated with the pressure $12 \mathrm{~atm}$. OCT 14 days after implantation.

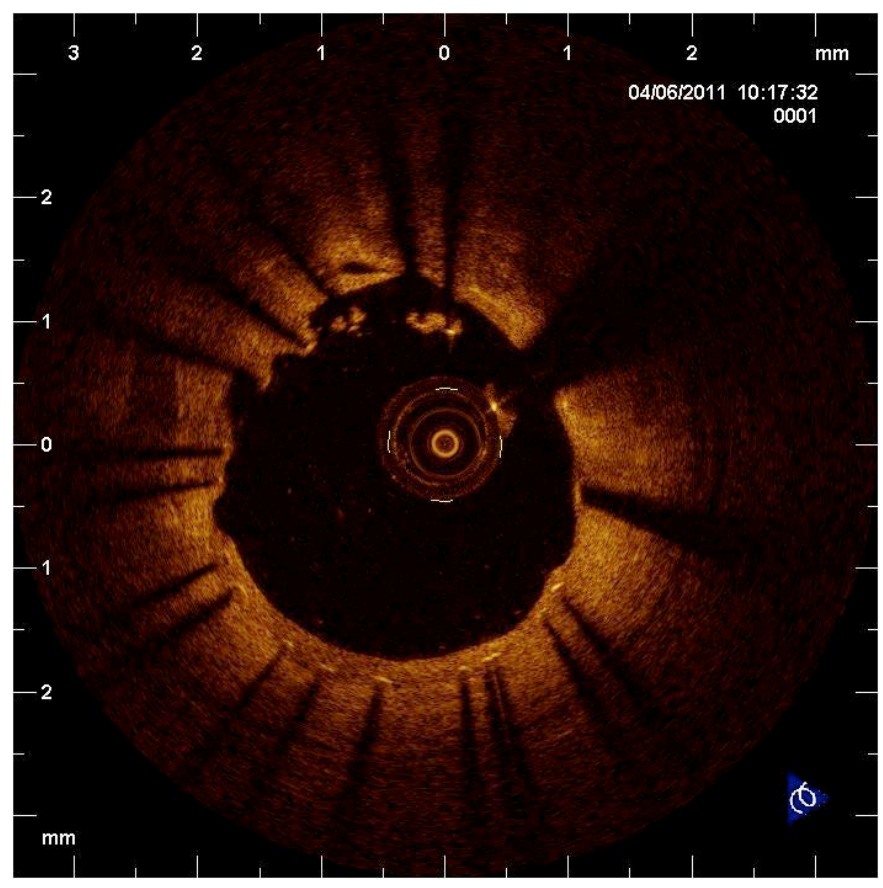

FIGURE 4. Stent with rapid endothelialization $3.0 \mathrm{~mm} 18 \mathrm{~mm}$ postdilated with noncompliant balloon catheter $3.5 \mathrm{~mm} 14 \mathrm{~mm}$ to 16 atm. The distal edge of the stent, where stent was postdilated, after 14 days. We see a very good healing of the stent in segments $1-9$ and poor apposition of the stent in 9-1 segments.

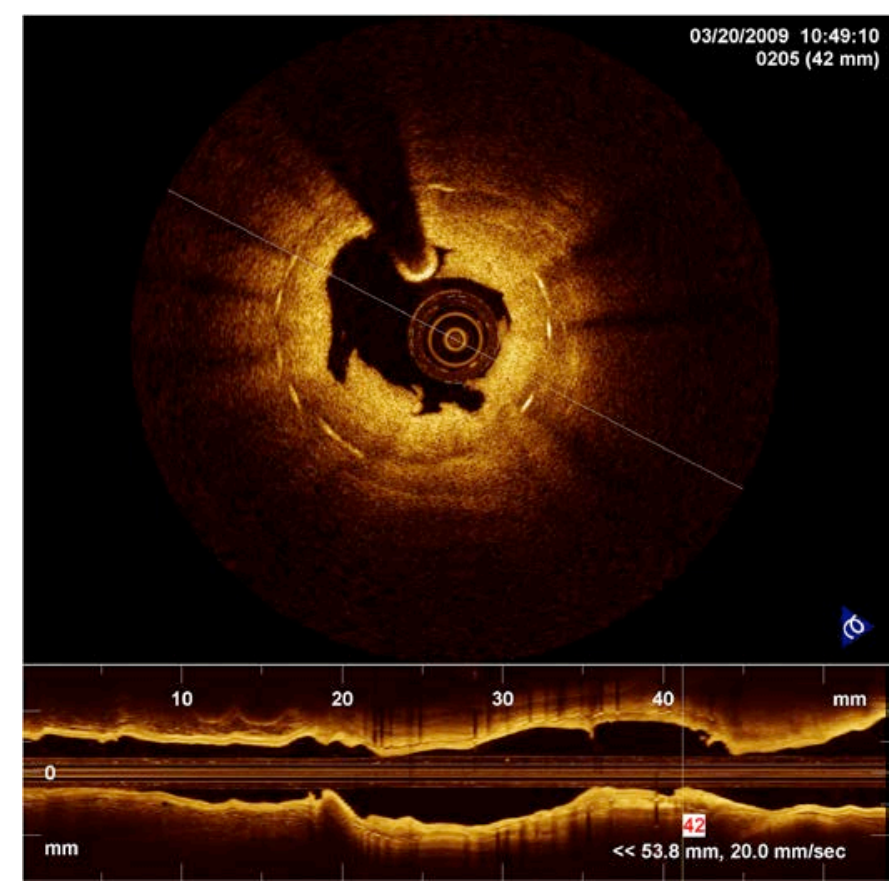

FIGURE 6. Patient with stent restenosis treated by balloon dilatation. 


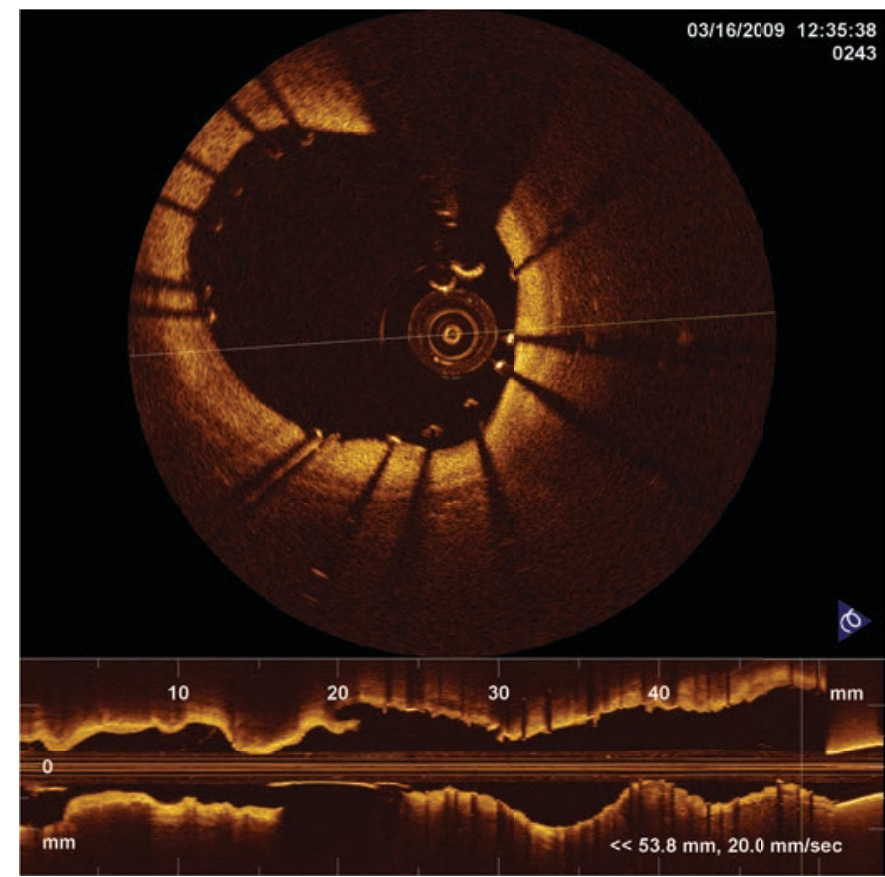

FIGURE 7. Malapposition of drug eluting stent (segments $3-6$, 9-12). OCT 14 days after implantation.

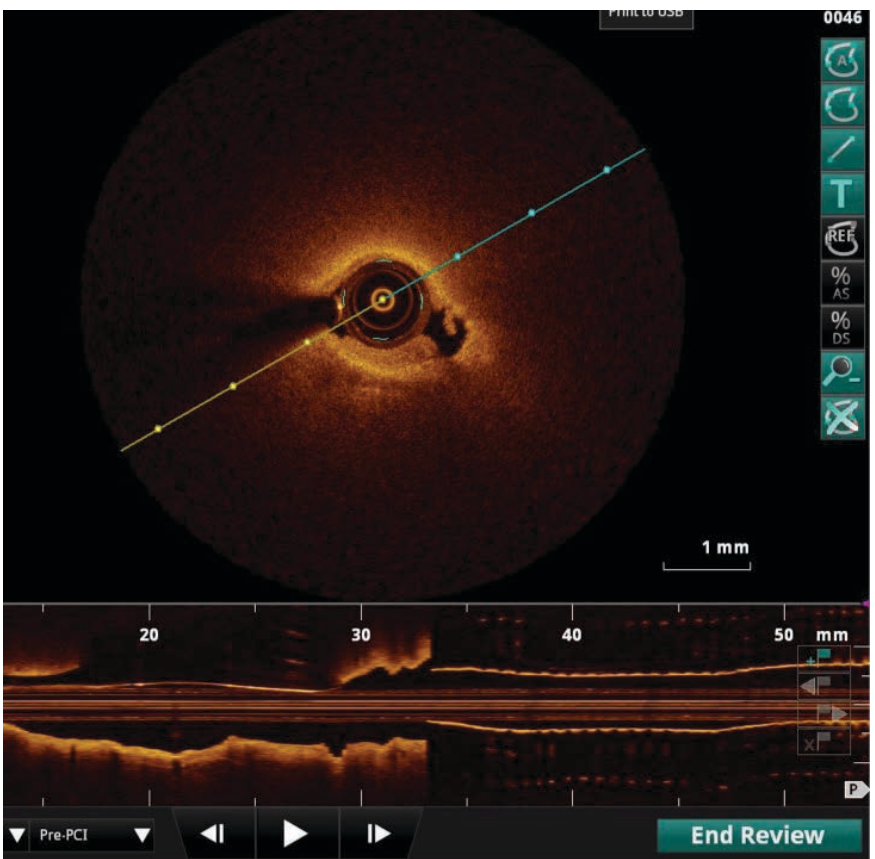

FIGURE 9. Stent thrombosis caused by neoatherogenesis 26 months after BMS implantation.

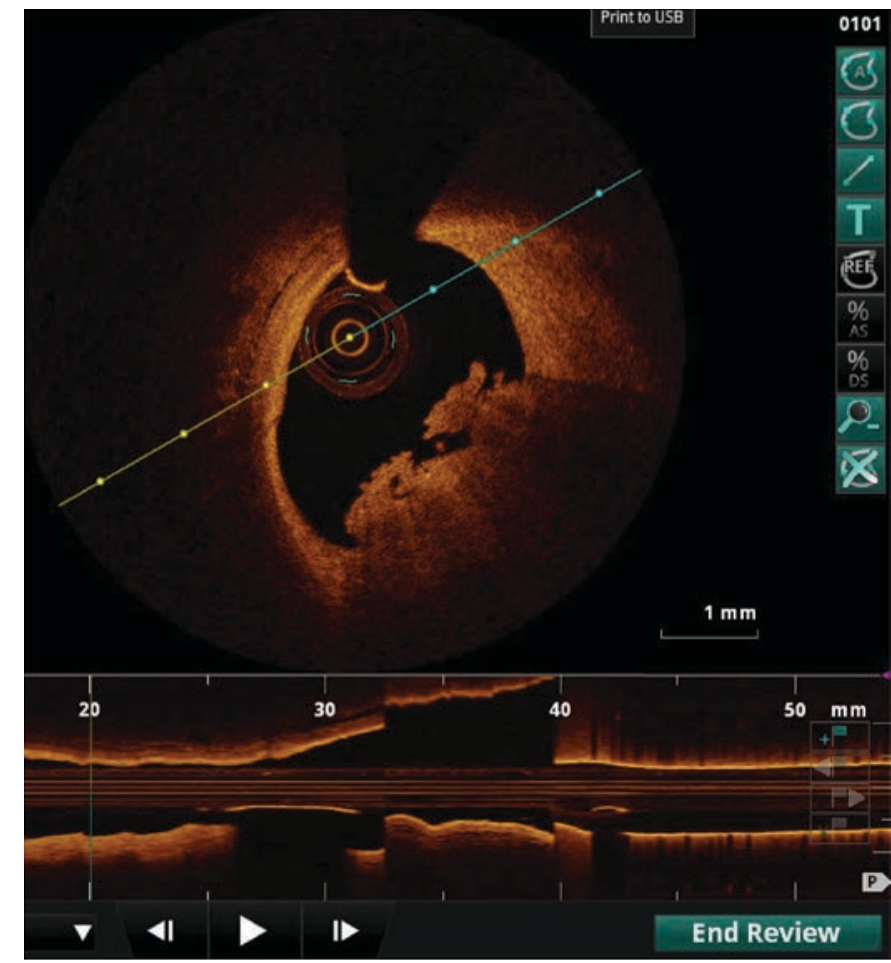

FIGURE 8. Thrombosis of proximal LAD causing acute coronary syndrome. Segments 3-6.

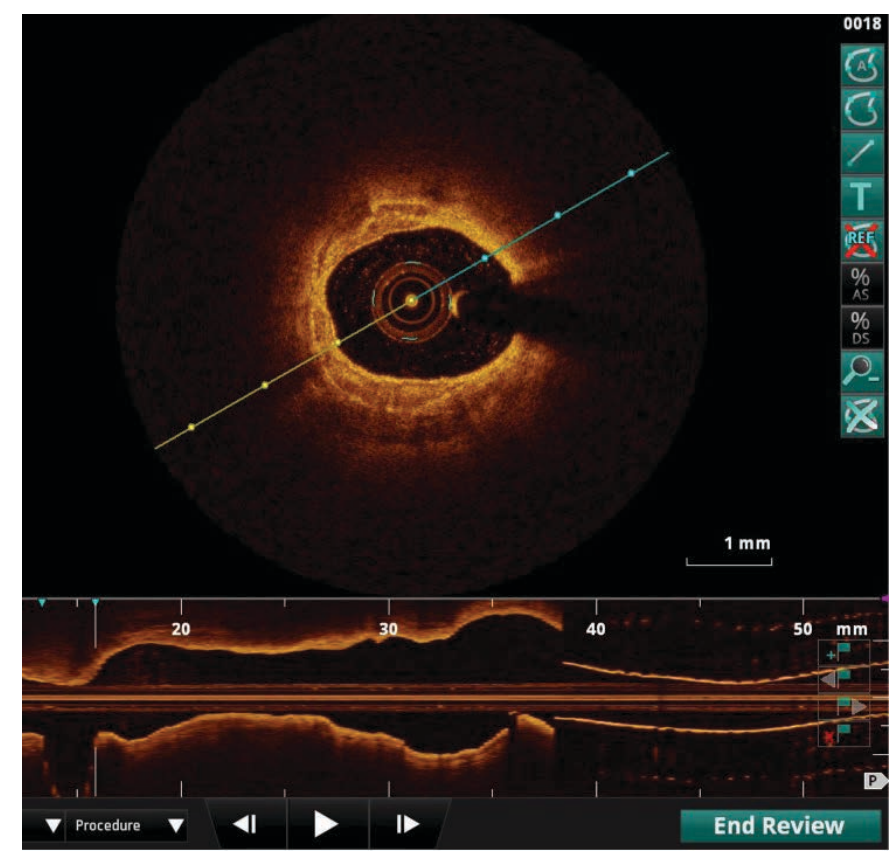

FIGURE 10. Fibrous atherosclerotic plaques in 84 years old female. 


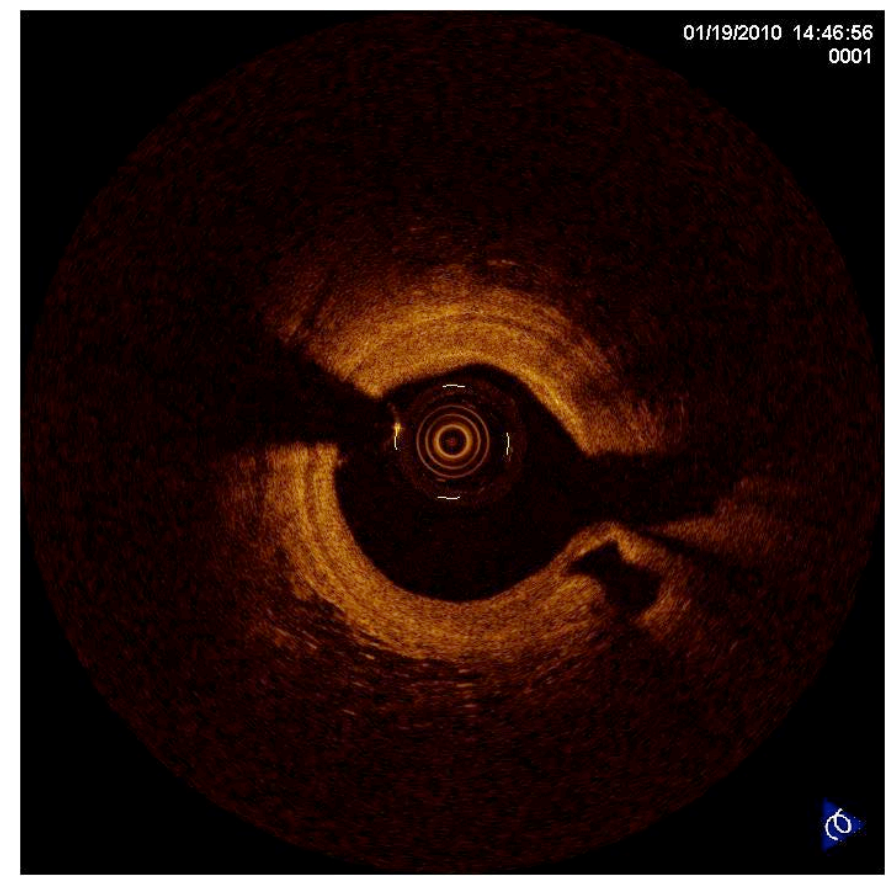

FIGURE 11. 64 years old patient 4 years after heart transplantation. Diffuse coronary disease. Intima is clearly thickened.

\section{CLINICAL TRIALS EVALUATING STENT IMPLANTATION AND STENT HEALING}

Clinical trials evaluating stent implantation and stent healing can be divided into two groups:

\section{TRIALS EVALUATING THE IMMEDIATE STATUS OF STENT IMPLANTATION}

IVUS studies have shown that stent malaposition is relatively rare and occurs in approximately $7 \%$ of the implanted stent struts [5]. Negative effect of stent malapposition on patients' prognosis was not found in IVUS trials. OCT is much more accurate than IVUS in assessment of interactions between the stent and the vessel wall. This is the reason why OCT diagnoses stent struts malapposition more often, up to $10 \%$ [6]. The risk factors are overlapping stents, stent length, complex lesions, strut thickness, stent design and acute stent recoil. Clinical consequences of stent malapposition diagnosed by OCT are not known. OCT can also describe well the possible damage of vascular wall by implanted stent, which is represented mainly by dissection at the edges of the stent. Protrusion of atherosclerotic plaque or thrombus through stent struts are observed quite often. Prognostic significance of these findings is unclear [7-13].

\section{TRIALS EVALUATING STENT HEALING AND STENT COVERAGE}

There are many reports on the topic of stent healing and their number grows rapidly. Unfortunately, prognostic data are missing. We do not know what percentage of uncovered stent struts counts for a real risk of stent thrombosis. We know from the pathological study done by Finn $\mathrm{AV}$ et al., that patients have 9 times higher risk of late stent thrombosis if the presence of uncovered stent struts exceeds 30\% [14]. Other studies compared stent healing among different types of stents at the same time after implantation [15-19]. Others studied stent healing of the same stent at different time intervals after implantation [20]. Kubo T et al. found that healing of drug eluting stents in patients with unstable angina pectoris is delayed in comparison with those who are stable [21]. Blinded evaluation, which means that the evaluator does not know the type of stent or clinical situation is very important from a methodological perspective. Independent core laboratories should be used for these measurements. "Uncovered" stent strut is defined as the absence of tissue on the stent strut. It should be noted that the resolution of OCT is $\geq 15 \mu \mathrm{m}$, therefore the presence of a single layer of endothelial cells on the stent strut may not be seen. On the other hand, the presence of tissue on the strut does not necessarily mean the presence of functional endothelium $[4,16]$. This is shown in the study done by Templin, who found differences in the optical density of fibrin and neointima that cover the stent [22]. Stent malapposition is diagnosed if we find a clear separation $\geq 150-200 \mu \mathrm{m}$ between the vessel wall and the stent strut [23]. We measure the distance between the inner edge of the strut and the vessel wall plus 20-30 $\mu \mathrm{m}$, which corrects the presence of artifacts and takes into account the axial resolution of OCT. There is no major sense to determine the coverage of malapposed struts, because they are prothrombogenic due to changes of blood flow, and because it is difficult to realize whether they are covered with fibrin or functional endothelium. All malapposed struts are regarded as potentially dangerous. Higher sensitivity for detection of neointimal hyperplasia by OCT compared to IVUS six months after implantation of bare metal and drug eluting stents was demonstrated in the Odessa trial [24]. The authors found that $92.7 \%$ of stent struts were covered with tissue, $5 \%$ were not covered and $2.3 \%$ were malapposed. No neointimal hyperplasia was seen by IVUS. The smallest strut coverage was observed in overlapping paclitaxel stents. Katoh et al. [25] found that almost 90\% of sirolimus stent struts were covered after 6 months and 
this percentage was still increasing in the next 6 months. Similar conclusions were reached by Li S et al. [15]), who found a lower prevalence of uncovered and malapposed struts of zotarolimus stents compared with sirolimus and paclitaxel stents $(3.0 \%$ vs $9.0 \%$ vs $6.2 \% ; 1.7 \%$ vs $6.4 \%$ vs $2.3 \%$ ) using OCT examination 12 months after implantation. The ENDEAVOR OCT study showed a low incidence of zotarolimus stent malapposition both immediately and 3 months after implantation $(6.0 \%$ vs $0.2 \%)[26,27]$. The LEADERS trial was a randomized, multicenter study comparing healing and malapposition of biolimus stent with a biodegradable polymer to sirolimus stent with permanent polymer. The authors discovered that the percentage of uncovered struts was lower in the biolimus group [28]. HORIZONS-OCT was a randomized study evaluating the stent coverage 13 months after acute myocardial infarction. Patients with paclitaxel stents had worse coverage but lower neointimal proliferation compared to bare metal stents [29]. The OCTAMI trial compared the coverage and malapposition of zotarolimus eluting and bare metal stents. No difference between both groups was found [30]. There is no significant difference between OCT and IVUS in the assessment of excessive neointimal proliferation associated with development of restenosis.

A large number of projects are underway. Rating of stent strut coverage after a certain period of time becomes an integral part of the development of new stents. The aim is that the new stent should have the best and fastest healing, minimal malapposition and small neointimal proliferation. The ABSORB EXTEND study, which follows the healing of everolimus eluting biodegradable stent is underway [31]. The OCT-EVEREST trial evaluates the healing of everolimus stents 6 months after implantation. Other ongoing trials in this field are CATANIA BRNO trial, TEST6-OCT trial, STACCATO trial, and COVER OCT-II. The OCTOPAS study uses OCT to assess the therapeutic effect of predilatation with paclitaxel eluting balloon (DEB) before implantation of a metal stent. The great European project PRESTIGE helps to uncover the causes of stent thrombosis. The Massachusetts General Hospital OCT Registry will provide us with information about the relationship between the nature of the atherosclerotic plaque and longterm prognosis [32].

\section{NEAR INFRARED SPECTROSCOPY}

The TVC Imaging System ${ }^{\mathrm{TM}}$, produced by Infraredx company, is a first-in-class intravascular imaging system with the unique ability to assess vessel composition and structure via integrated near infrared spectroscopy (NIRS) lipid

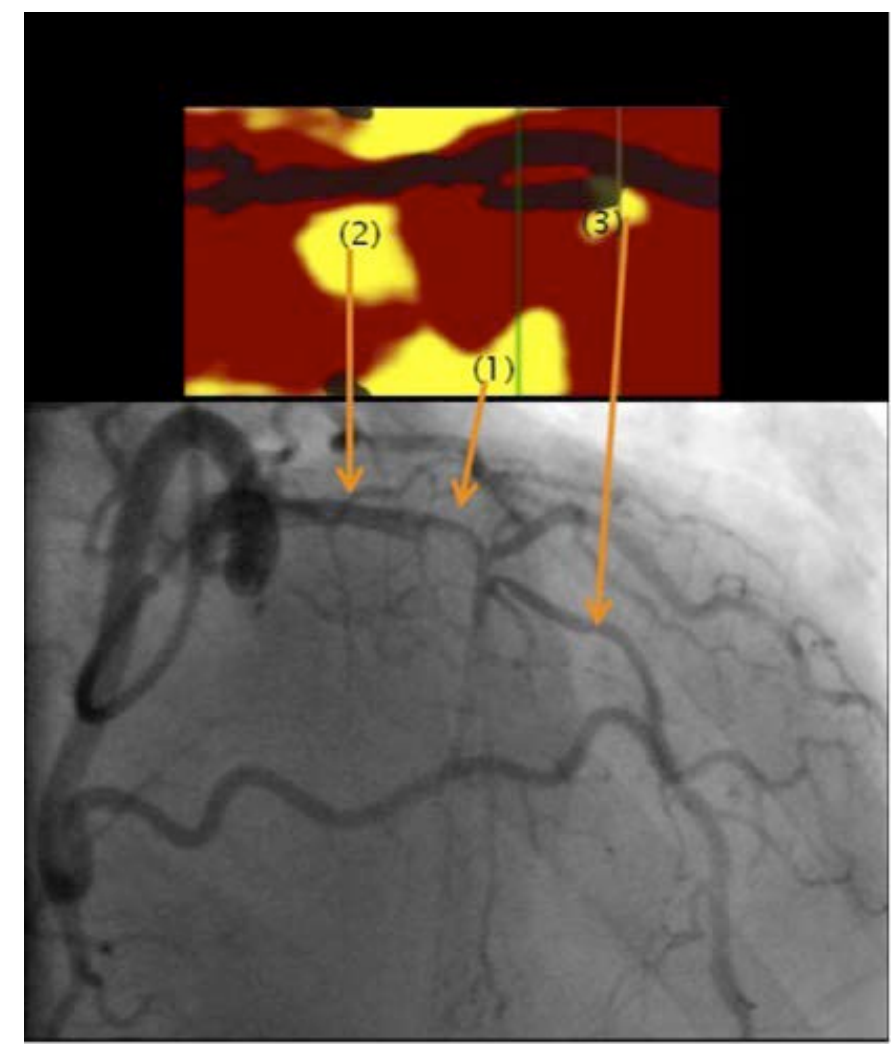

FIGURE 12. NIRS (Chemogram ${ }^{T M}$ ) and coronary angiography of a patient with acute coronary syndrome. (1) Abundant lipid core at culprit site. (2) Additional large lipid core proximal to cuprit lesion. (3) Small lipid core distal to culprit. Stenting from (2) to (1) is recommended.

core plaque detection and enhanced IVUS imaging technology [33]. The TVC Imaging System ${ }^{\mathrm{TM}}$ has one console and one catheter for imaging, which works in two modalities during pullback. Near infrared spectroscopy uses near infrared laser light, which is directed at the vessel wall from the rotating catheter core. Light reflected from the lesion is detected by a sensor. During pullback and rotation, 40 spectra are acquired per second. Each spectrum provides data on the chemical content of a single, small area of the vessel wall. The spectra are analyzed using an algorithm validated in autopsy specimens that indicates the chemical composition of the plaque. The algorithm is trained to indicate the probability of lipid core at each pixel and a visual representation of the spectroscopic data, called a Chemogram $^{\mathrm{TM}}$, is generated. The Chemogram ${ }^{\mathrm{TM}}$ provides a detailed map of presence or absence of lipid core in the arterial wall. Studies in human autopsy specimens have proven that the TVC Imaging System ${ }^{\mathrm{TM}}$ can identify lipid core plaque. The TVC Imaging System ${ }^{\mathrm{TM}}$ utilizes a single catheter that emits both a near infrared laser beam and a high frequency ultrasound beam. Both types of imaging data are collected simultaneously in real time. A TVC Com- 
posite Image is produced. Both IVUS cross sectional and longitudinal views are displayed and the Chemogram ${ }^{\mathrm{TM}}$ is shown. A slice from the Chemogram ${ }^{\mathrm{TM}}$ showing lipid core data is shown as a halo around the corresponding IVUS cross section. A block Chemogram ${ }^{\mathrm{TM}}$ is provided, spatially co-registered with the longitudinal view IVUS. This means that by utilizing NIRS, the TVC Imaging System enables the immediate, accurate, and reliable identification and location of lipid core plaque known to complicate stenting, as well as quantification of the vessel's lipid core burden. Through IVUS, the TVC Imaging System provides clear and relevant information about vessel structure, including lumen dimensions, degree of stenosis, and plaque burden. It is the first and only FDA-cleared device for the detection of lipid core plaques of interest. TVC-guided stenting holds the potential to minimize procedural complications and possibly to prevent periprocedural myocardial infarction.

There is only a limited but rapidly increasing number of clinical trials using NIRS. Dixon et al. showed that lipid core plaque extending into and beyond the intended stent margine has implications for stent length selection and optimal lesion coverage [34]. Lipid core burden index calculated by software for $4 \mathrm{~mm}$ length of coronary artery (maxLCBI/mm) higher or equal 500 has a direct relation to the risk of periprocedural myocardial infarction during stenting [35-37]. Several important prognostic trials like Prospect and Lipid-rich plaque (LRP) study using detection of lipid core plaque as a marker for subsequent clinical event are ongoing $[31,38]$.

\section{CONCLUSION}

Optical coherence tomography is a rapidly developing method. It provides unreplaceable information about the inner structures of coronary wall, about the presence, extent and risk of atherosclerotic plaques, the quality of stent implantation and stent healing. There is a great hope that OCT-based indicators of patient's prognosis will be defined soon. The TVC Imaging System ${ }^{\mathrm{TM}}$ assesses vessel composition and structure via integrated near infrared spectroscopy lipid core plaque detection and enhanced IVUS imaging technology. It provides clear and relevant information about vessel structure, including lumen dimensions, degree of stenosis, and plaque burden. It is the first and only FDA-cleared device for the detection of lipid core plaques which has a potential for important prognostic information. It can minimize procedural complications and possibly prevent periprocedural myocardial infarction. There is no doubt that new imaging modalities have a great future in interventional cardiology.

\section{ACKNOWLEDGEMENT}

This study was supported by Research Intention of Ministry of Education of Czech Republic (MSM 0021622402), by Research Grant IGA MZ ČR NT11412-5/2010 and European Regional Development Fund - Project FNUSA-ICRC (CZ.1.05/1.1.00/02.0123)

\section{REFERENCES}

1. Huang D, Swanson EA, Lin CP, et al. Optical coherent tomography. Science. 1991;254:1178-1181.

2. Prati F, Regar E, Mintz GS, et al. Imaging of atherosclerosis: optical coherence tomography (OCT). Expert review document on methodology, terminology, and clinical applications of optical coherence tomography: physical principles, methodology of image acquisition, and clinical application for assessment of coronary arteries and atherosclerosis. Eur Heart J 2010; 31:401-415.

3. Špaček R, Červinka P. Nové možnosti zobrazení koronárních tepen - optická koherentní tomografie. Cor Vasa. 2009;Supplementum 1:43-46.

4. Regar E, Ligthart J, Bruining N, van Soest G. The diagnostic value of intracoronary optical coherence tomography. Herz. 2011;36:417-429.

5. Hong MK, Mintz GS, Lee CW et al. Late stent malaposition after drug-eluting stent implantation: an intravascular ultrasound analysis with long-term follow-up. Circulation. 2006;113:414-419.

6. Tanigawa J, Barlis P, Kaplan S, Goktekin OCD. Stent strup apposition in complex lesions using optical coherence tomography. Am J Cardiol. 2006;98(Suppl 1):97M.

7. Radu M, Jørgensen E, Kelbæk H, Helqvist S, Skovgaard L, Saunamäki K. Optical coherence tomography at followup after percutaneous coronary intervention: relationship between procedural dissections, stent strut malapposition and stent healing. EuroIntervention. 2011;7:353-361.

8. Reimers B, Nikas D, Stabile E, Favero L, Saccà S, Cremonesi A, Rubino P. Preliminary experience with optical coherence tomography imaging to evaluate carotid artery stents: safety, feasibility and techniques. EuroIntervention. 2011;7:98-105.

9. Liu Y, Imanishi T, Kubo T, et al. Assessment by optical coherence tomography of stent struts across side branch. Comparison of bare-metal stents and drug-elution stents. Circ J. 2010;75:106-112.

10. Murata A, Wallace-Bradley D, Tellez A, et al. Accuracy of optical coherent tomography in the evaluation of neointimal coverage after stent implantation. JACC Cardiovasc Imaging. 2010;3:76-84.

11. Suzuki Y, Ikeno F, Koizumi T, et al. In vivo comparison between optical coherent tomography and intravascular ultrasound for detecting small degrese of in-stent neointima after stent implantation. JACC Cardiovasc Interv. 2008;1:168-173.

12. Capodanno D, Prati F, Pawlowsky T, Cera M, La Manna A, Albertucci M, Tamburino C. Comparison of optical coherence tomography and intravascular ultrasound for the assessment of in-stent tissue coverage after stent implantation. EuroIntervention. 2009;5:538-543. 
13. www.medis.nl

14. Finn AV, Joner M, Nakazawa G, et al. Pathological correlates of late drug-eluting stent thrombosis: strut coverage as a marker of endothelization. Circulation. 2007;115:24352441.

15. Li S, Wang Y, Gai L, et al. Evaluation of neointimal coverage and apposition with various drug-eluting stents over 12 months after implantation by optical coherence tomography. Int J Cardiol. 2011;doi:10.1016/j.ijcard.2011.05.076.

16. Fujii K, Kawasaki D, Oka K, et al. Endothelium-dependent coronary vasomotor response and neointimal coverage of zotarolimus-eluting stents 3 months after implantation. Heart. 2011;97:977-82.

17. Inoue $\mathrm{T}$, Shite $\mathrm{J}$, Yoon $\mathrm{J}$, et al. Optical coherence evaluation of everolimus-eluting stents 8 months after implantation. Heart. 2011;97:1379-1384.

18. Guagliumi G, Sirbu V, Musumeci G, et al. Strut coverage and vessel wall response to a new-generation paclitaxeleluting stent with an ultrathin biodegradable abluminal polymer: Optical Coherence Tomography Drug-Eluting Stent Investigation (OCTDESI). Circ Cardiovasc Interv. 2010;3:367375.

19. La Manna A, Capodanno D, Cera M, et al. Optical coherence tomographic results at six-month follow-up evaluation of the CATANIA coronary stent system with nanothin Polyzene-F surface modification (from the Assessment of The LAtest Non-Thrombogenic Angioplasty Stent [ATLANTA] trial). Am J Cardiol. 2009;103:1551-1555.

20. La Manna A, Prati F, Capodanno D, et al. Head-to-head comparison of early vessel healing by optical coherence tomography after implantation of different stents in the same patient. J Cardiovasc Med. 2011;12:328-333.

21. Kubo $\mathrm{T}$, Imanishi $\mathrm{T}$, Kitabata $\mathrm{H}$., et al. Comparison of vascular response after sirolimus-eluting stent implantation between patients with unstable and stable angina pectoris: a serial optical coherence tomography study. JACC Cardiovasc Imaging. 2008;1:475-484.

22. Templin C, Meyer M, Muller MF, et al. Coronary optical frequency domain imaging (OFDI) for in vivo evaluation of stent healing: comparison with light and electron microscopy. Eur Heart J. 2010;31:1792-1801.

23. Tahara S, Bezerra HG, Sirbu V, et al. Angiographic, IVUS and OCT evaluation of the long-term impact of coronary disease severity at the site of overlapping drug-eluting and bare metal stents: a substudy of the ODESSA trial. Heart. 2010;96:15741578 .

24. Guagliumi G, Musumeci G, Sirbu V, et al. Optical coherence tomography assessment of in vivo vascular response following implantation of overlapping bare-metal and drug-eluting stents. JACC Cardiovasc Interv. 2010;3:531-539.
25. Katoh H, Shite J, Shinke T, et al. Delayed neontimalization on sirolimus-eluting stents: 6-month and 12-month follow up by optical coherence tomography. Circ J. 2009;73:1033-1037.

26. Kim JS, Jang IK, Fan C, et al. Evaluation in 3 months duration of neointimal coverage after zotarolimus-eluting stent implantation by optical coherence tomography: the ENDEAVOR OCT trial. JACC Cardiovasc Interv. 2009;2:12401247.

27. Kim JS, Fan C, Choi D, et al. Different patterns of neointimal coverage between acute coronary syndrome and stable angina after various types of drug-eluting stents implantation; 9-month follow-up optical coherence tomography study. Int J Cardiol. 2011;146:341-346.

28. Barlis P, Regar E, Serruys PW, et al. An optical coherence tomography study of a biodegradable vs durable polymercoated limus-eluting stent: a LEADERS trial substudy. Eur Heart J. 2010;31:165-176.

29. Guagliumi G, Sirbu V, Costa MS, et al. Long-term strut coverage of paclitaxel eluting stents compared to bare-metal stents implanted during primary PCI in acute myocardial infarction: HORIZONS-OCT. Circulation. 2011;123:274-281.

30. Guagliumi G, Sirbu V, Bezerra HG, et al. Strut coverage and vessel wall response to zotarolimus-eluting and bare metal stents implanted in patients with ST-elevation myocardial infarction: the OCTAMI study. JACC Cardiovasc Interv. 2010;3:680-687.

31. www.clinicaltrials.gov

32. Tahara S, Chamié D, Baibars M, Alraies C, Costa M. Optical coherence tomography endpoints in stent clinical investigations: strut coverage. Int J Cardiovasc Imaging. 2011;27:271-287.

33. www.infraredx.com

34. Dixon SR, Grines CL, Munir A et al. Analysis of target lesion length before coronary artery stenting using angiography and near-infrared spectroscopy versus angiography alone. Am J Cardiol. 2012;109:60-66.

35. Goldstein JA, Maini B, Dixon SR et al. Detection of lipidcore plaques by intracoronary near-infrared spectroscopy identifies high risk of periprocedural myocardial infarction. Circ Cardiovasc Interv. 2011;4:429-437.

36. Groves EM, Seto AH, Kern MJ. Invasive Testing for Coronary Artery Disease: FFR, IVUS, OCT, NIRS. Cardiol Clin. 2014;32:405-417.

37. Roleder T, Kovacic JC, Ali Z et al. Combined NIRS and IVUS imaging detects vulnerable plaque using a single catheter system: a head-to-head comparison with OCT. EuroIntervention. 2014;10:303-311.

38. Brugaletta S, Sabaté M. Assessment of plaque composition by intravascular ultrasound and near-infrared spectroscopy: from PROSPECT I to PROSPECT II. Circ J. 2014;78:1531-1539. 